\title{
Ambiente construído e bem-estar subjetivo: análise das diferenças entre os usuários dos diferentes modos de transporte
}

\author{
Ariadne Amanda Barbosa Samios ${ }^{1}$, Ana Margarita Larranaga ${ }^{2}$, Helena Bettella Cybis ${ }^{3}$ \\ 1WRI Brasil, ariadneabs@gmail.com \\ 2Programa de Pós-graduação em Engenharia de Produção, UFRGS, analarra@producao.ufrgs.br \\ ${ }^{3}$ Programa de Pós-graduação em Engenharia de Produção, UFRGS, helenabc@producao.ufrgs.br
}

\section{Recebido:}

19 de abril de 2019

Aceito para publicação:

4 de outubro de 2019

Publicado:

12 de novembro de 2019

Editor de área:

Cira Pitombo

\section{Palavras-chaves:}

Caminhabilidade,

Modelos de equações estruturais,

Segurança pública,

Percepção do bairro,

Satisfação com o bairro.

\section{Keywords:}

Walkability,

Structural equation modelling,

Security,

Neighborhood perception,

Neighborhood satisfaction.

DOI:10.14295/transportes.v27i3.2021

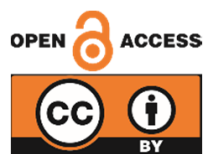

\begin{abstract}
RESUMO
O bem-estar subjetivo (subjective well-being) tem sido foco de crescente atenção na comunidade acadêmica nas últimas décadas, e especificamente na área de transportes (economia, ambiente urbano, mobilidade e acessibilidade). Os objetivos desse estudo são analisar a existência de diferenças na percepção do ambiente construído entre usuários de transporte ativo, transporte coletivo e transporte individual; e comparar o efeito do ambiente construído no bem-estar para os três grupos de usuários. Utilizando dados de uma pesquisa de atividade física na cidade de São Paulo - Brasil, modelos de equações estruturais multigrupo foram estimados. $O$ estudo mostrou que o ambiente construído influencia o bem-estar dos indivíduos para os três grupos de usuários. Evidenciou a existência de diferenças na percepção do ambiente construído entre os grupos. Os resultados podem contribuir para debates acadêmicos e políticos sobre bemestar, qualidade de vida e sustentabilidade urbana considerando a falta de investigações empíricas aprofundadas sobre este tópico.
\end{abstract}

\begin{abstract}
Subjective well-being has been focus of increased attention in the research community during the last decades and specifically in the transportation field (economy, urban environment, mobility and accessibility). The purposes of this study are to analyze the existence of differentiated perceptions of the built environment among users of active travel, public and private transportation; and comparing the effect of built environment on well-being for the three groups of users. Using data from a physical activity survey of São Paulo - Brazil, multi-group structural equation models were estimated. The study showed that the built environment influences the individual's well-being for the three groups of users. It evidenced the existence of differences in built environment perceptions between the groups. The results could contribute to academic and policy debates on well-being, quality of life and urban sustainability considering a lack of empirical research on this topic.
\end{abstract}

\section{INTRODUCÃO}

O bem-estar subjetivo (BES) (subjective well-being - SWB) tem sido foco de crescente atenção na comunidade acadêmica nas últimas décadas. Estudos nessa área utilizam diversas denominações, tais como: felicidade, satisfação, afeto positivo e avaliação subjetiva da qualidade de vida (Joshanloo, 2017; Heizomi et al. 2015; Musa et al. 2018). Embora existam várias definições de bem-estar subjetivo, a mais utilizada é a proposta por Diener (1984), na qual o bem-estar subjetivo refere-se à experiência individual e subjetiva da avaliação da vida como positiva, e inclui variáveis como satisfação com a vida e vivência de afeto positivo (Diener,1984; 1994). Assim, BES refere-se a como as pessoas se sentem e avaliam suas vidas. 
$\mathrm{Na}$ área de transportes, os estudos se concentram em identificar formas pelas quais o transporte pode afetar o bem-estar da população, através da economia, ambiente urbano, mobilidade e acessibilidade (Spinney et al., 2009; Delbosc, 2012; Reardon e Abdallah, 2013; Singleton, 2018). Vários estudos mostraram que a mobilidade está positivamente associada com o bemestar e a qualidade de vida. Trabalhos recentes sugerem que a melhoria do bem-estar, e não a melhoria da mobilidade ou acessibilidade deveria ser o objetivo final da política de transportes (Stanley e Stanley, 2007; Stanley et al., 2011). Sem esse objetivo em mente, a política de transportes pode acabar incentivando apenas a mobilidade per se, aumentando as externalidades associadas ao congestionamento e ao meio ambiente, sem impacto em resultados específicos de bem-estar (Stanley e Vella-Brodrick, 2009; Stanley e Stanley, 2007; Stanley et al., 2011).

Nos últimos anos, pesquisadores têm prestado crescente atenção na relação entre ambiente construído e bem-estar, desenvolvendo diversos estudos nessa linha de pesquisa (Leyden, 2003; Ziersch et al., 2005; Hipp e Perrin, 2009; Wood et al., 2010; Cabrera, 2013, Mouratidis, 2018). Pesquisas sugerem que formas urbanas compactas, caracterizadas por uso do solo heterogêneo, alta densidade residencial, alta acessibilidade e oferta de transporte público aumentam o bem-estar geral dos indivíduos, criando mais oportunidades de sociabilização (Mouratidis, 2018).

Embora alguns estudos tenham surgido na literatura internacional, a relação entre estrutura urbana e BES tem sido objeto de estudo recente e a relação ainda não está estabelecida. Adicionalmente, os estudos existentes não examinaram como os efeitos do ambiente construído podem diferir entre os usuários de diferentes modos de transporte. 0 bem-estar varia com características dos indivíduos tais como gênero (Elias et al., 2015; Basarić et al., 2016), renda (Wang e Cao, 2017; Lin et al., 2017), características culturais (Hu, 2017), percepções (González et al., 2015) e posição familiar (Yang et al., 2017). Assim, o efeito do ambiente construído no bemestar também pode variar. 0 poder de políticas urbanas que visem melhorar o bem-estar da população pode ser desigual e até ineficiente para alguns grupos de usuários. A distinção entre os tipos de usuários pode ser especialmente importante em países como o Brasil, onde características socioeconômicas estão fortemente ligadas com a escolha modal (Larranaga et al., 2009; Larranaga e Cybis, 2014).

Esse estudo visa dois objetivos. Primeiro, analisar a existência de diferenças na percepção do ambiente construído entre usuários de transporte ativo (TA), coletivo (TC) e individual (TI). Segundo, comparar o efeito do ambiente construído no bem-estar para os três grupos de usuários. Para isto, foi realizado um estudo de caso em uma região da cidade de São Paulo, Brasil, através de modelagem de equações estruturais multigrupo. A técnica adotada permite analisar as inter-relações entre as diferentes características estudadas e comparar a invariância das relações, estruturas e parâmetros em cada um dos grupos analisados.

\section{COLETA DE DADOS}

\subsection{Amostra e região pesquisada}

Os dados utilizados são provenientes de uma pesquisa de entrevistas domiciliares sobre nível de atividade física, conduzida e disponibilizada, aos autores, pelo WRI Brasil (World Resources Institute Brasil), organização de pesquisa internacional, sem fins lucrativos. A área de estudo foi definida através do local de aplicação do questionário, o qual originalmente foi aplicado para avaliar os impactos da implantação do corredor de BRT (Bus Rapid System) Radial Leste em São 
Paulo. A região pesquisada compreendeu parte dos bairros Tatuapé, Carrão, Penha e Vila Matilde, localizados na zona leste de São Paulo, abrangendo o entorno (500 m) das estações de metrô presentes nas áreas de Tatuapé, Carrão e Penha.

As proximidades das três estações apresentam características heterogêneas em relação ao uso do solo. Quadras com construções residenciais de médio ou alto padrão, assim como quadras de uso misto, com residências, comércios e indústrias, podem ser observadas no entorno da estação Penha. Quadras residenciais, de médio e alto padrão, também estão presentes no entorno da estação Carrão, mas surgem algumas quadras com comércios e serviços de pequeno a médio porte e uma área de lazer de maior porte, Parque Sampaio. 0 entorno da estação Tatuapé apresenta a maior concentração de quadras com uso misto. Esta área possui características comerciais mais consolidadas, com estabelecimentos comerciais de diversos portes além do Shopping Tatuapé, o qual possui uma conexão direta com a estação Tatuapé.

0 banco de dados consiste em 2300 entrevistas individuais e domiciliares realizadas entre agosto e setembro de 2015. A seleção da amostra foi baseada em um processo de amostragem probabilístico por conglomerados de múltiplos estágios. Aleatoriamente, foram sorteados os quarteirões, ruas e as casas ou apartamentos dentro da área de estudo. Para a seleção do morador a ser entrevistado, foi utilizado o critério de data de aniversário mais próxima à realização da entrevista. A taxa de recusa foi de 6\% e a de desistência de 4\%. Foram entrevistadas pessoas pertencentes à faixa etária de 18 a 70 anos, alfabetizadas, que não possuíam limitações cognitivas nem mobilidade reduzida e que residiam no mínimo há um ano na área pesquisada. A Figura 1 apresenta a localização das entrevistas na área de estudo.

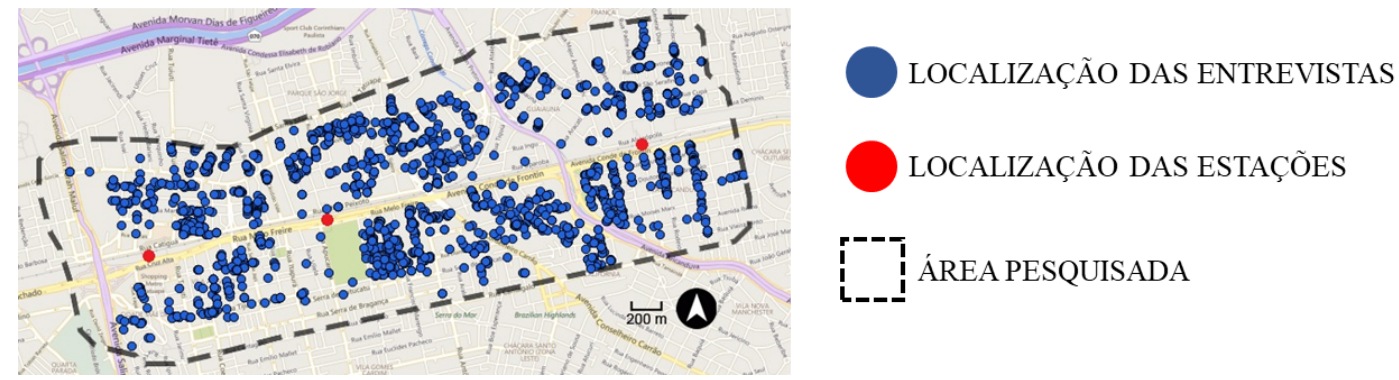

Figura 1. Localização das entrevistas realizadas e das três estações

\subsection{Questionário}

As entrevistas foram realizadas com questionário pré-estruturado com tempo de aplicação médio de 15 minutos. 0 questionário foi estruturado em sete partes: (i) Filtros, (ii) Dados do entrevistado, (iii) Meios de transporte, (iv) Atividade física de deslocamento, (v) Atividade física no tempo de lazer, (vi) Percepções do ambiente no bairro e (vii) Qualidade de vida.

A primeira parte do questionário abrangia perguntas de controle para realizar a entrevista completa apenas com pessoas que se enquadravam nos critérios de faixa etária e tempo de residência no bairro. A segunda parte continha perguntas sobre dados pessoais do entrevistado, tais como gênero, peso, altura, estado civil e escolaridade. A terceira parte abordava o padrão de viagem dos entrevistados e os modos de transporte utilizados. Os modos pesquisados foram caminhada, bicicleta, automóvel, motocicleta, taxi, ônibus, fretado, metrô (operado pela Companhia do Metropolitano de São Paulo) e trens metropolitanos (operados pela Companhia Paulista de Trens Metropolitanos). As perguntas eram estratificadas por modo e questionavam 
quantos dias a pessoa utilizava o modo de transporte específico para ir e voltar de algum local em viagens utilitárias. Essas perguntas eram complementadas por outras relativas ao tempo de caminhada antes de entrar e após sair do veículo, assim como o tempo gasto dentro do veículo.

A quarta e quinta parte do questionário continham perguntas relativas aos deslocamentos ativos e atividade física e questionavam sobre viagens realizadas na semana anterior por bicicleta ou a pé e com duração mínima de 10 minutos. Na quarta parte, os deslocamentos se referiam a viagens utilitárias a pé ou de bicicleta. Na quinta, os deslocamentos ativos se referiam a viagens não utilitárias, realizadas por recreação ou esporte.

Tabela 1 - Estatística descritiva dos dados utilizados

\begin{tabular}{|c|c|c|c|c|c|}
\hline & Mínimo & Máximo & Média & Desvio Padrão & Coef. De Variação \\
\hline \multicolumn{6}{|l|}{ Perfil dos entrevistados } \\
\hline Tempo_residência (anos) & 1 & 70 & 17,0 & 14,0 & 0,82 \\
\hline Idade (anos) & 18 & 70 & 40,6 & 16,0 & 0,39 \\
\hline \multicolumn{6}{|l|}{ Hábitos semanais de viagem } \\
\hline Caminhada_frequência semanal de viagens & 0 & 14 & 2,708 & 4,443 & 1,64 \\
\hline Caminhada_tempo semanal (min) & 0 & 1260 & 66,367 & 134,759 & 2,03 \\
\hline Caminhada_tempo semanal de acesso $(\mathrm{min})^{\mathrm{a}}$ & 0 & 720 & 105,360 & 108,287 & 1,03 \\
\hline Bicicleta_frequência semanal de viagens & 0 & 14 & 0,450 & 2,037 & 4,53 \\
\hline Bicicleta_tempo semanal (min) & 0 & 1400 & 14,732 & 84,462 & 5,73 \\
\hline Automóvel_frequência semanal de viagens & 0 & 14 & 3,062 & 4,710 & 1,54 \\
\hline Automóvel_tempo semanal (min) & 0 & 1820 & 115,603 & 209,552 & 1,81 \\
\hline Moto_frequência semanal de viagens & 0 & 14 & 0,925 & 3,086 & 3,34 \\
\hline Moto_tempo semanal (min) & 0 & 1820 & 30,945 & 121,289 & 3,92 \\
\hline Táxi_frequência semanal de viagens & 0 & 10 & 0,054 & 0,514 & 9,53 \\
\hline Táxi_tempo semanal (min) & 0 & 280 & 1,196 & 13,751 & 11,50 \\
\hline Ônibus_frequência semanal de viagens & 0 & 14 & 1,823 & 3,686 & 2,02 \\
\hline Ônibus_tempo semanal (min) & 0 & 1200 & 65,886 & 157,411 & 2,39 \\
\hline Fretado_frequencia semanal de viagens & 0 & 14 & 0,340 & 1,781 & 5,24 \\
\hline Fretado_tempo semanal (min) & 0 & 900 & 15,235 & 86,637 & 5,69 \\
\hline Metrô_frequência semanal de viagens & 0 & 14 & 1,698 & 3,695 & 2,18 \\
\hline Metrô_tempo semanal (min) & 0 & 1370 & 48,469 & 117,470 & 2,42 \\
\hline CPTM_frequência semanal de viagens & 0 & 14 & 0,186 & 1,261 & 6,78 \\
\hline CPTM_tempo semanal (min) & 0 & 450 & 4,495 & 35,147 & 7,82 \\
\hline Satisfação_bairro* (índice) & 1 & 4 & 2,825 & 0,588 & 0,21 \\
\hline Frequências de respostas (\%) & Valor 1 & Valor 2 & Valor 3 & Valor 4 & Valor 5 \\
\hline \multicolumn{6}{|l|}{ Percepções das características do bairro } \\
\hline Facil_acesso_TC ${ }^{b}$ & 6,2 & 14,8 & 31,1 & 47,9 & - \\
\hline Coisas_interessantes_ver ${ }^{b}$ & 8,9 & 27,2 & 35,5 & 28,4 & - \\
\hline Construçoes_casas_bonitas ${ }^{\mathrm{b}}$ & 3,3 & 20,9 & 40,3 & 35,5 & - \\
\hline Travessias_facilitadas ${ }^{\mathrm{b}}$ & 6,1 & 18,6 & 49,0 & 26,3 & - \\
\hline Canteiro_verde ${ }^{b}$ & 27,4 & 19,7 & 30,3 & 22,6 & - \\
\hline Seguro_TA_diab & 5,6 & 13,1 & 46,2 & 35,1 & - \\
\hline Seguro_TA_noite ${ }^{b}$ & 13,7 & 25,2 & 41,4 & 19,7 & - \\
\hline Seguro_embar_desembar_TC ${ }^{b}$ & 3,4 & 14,0 & 42,6 & 40,0 & - \\
\hline Seguro_parq_espaç_publicos ${ }^{b}$ & 4,7 & 14,3 & 36,8 & 44,2 & - \\
\hline \multicolumn{6}{|l|}{ Satisfação com o bairro } \\
\hline Satisf_acesso_TC ${ }^{c d}$ & 3,1 & 18,8 & 43,6 & 34,5 & - \\
\hline Satisf_calçadas ${ }^{c d}$ & 16,9 & 21,1 & 44,3 & 17,7 & - \\
\hline Satisf_espaço_bicicletas ${ }^{c d}$ & 15,9 & 17,9 & 39,5 & 26,7 & - \\
\hline Satisf_acesso_opçoes_lazer ${ }^{c d}$ & 6,0 & 25,4 & 40,3 & 28,3 & - \\
\hline Satisf_espaços_públicos_lazercd & 9,9 & 29,5 & 38,7 & 21,9 & - \\
\hline Satisf_acesso_comerciocd & 3,3 & 16,4 & 55,8 & 24,5 & - \\
\hline Satisf_segurança_publicacd & 14,4 & 26,7 & 44,2 & 14,7 & - \\
\hline Satisf_serviços_publicos ${ }^{c d}$ & 11,0 & 18,9 & 41,7 & 28,4 & - \\
\hline \multicolumn{6}{|l|}{ Qualidade de vida } \\
\hline Bem_estare & 1,8 & 3,9 & 13,3 & 47,0 & 34,0 \\
\hline $\begin{array}{l}\text { aSoma dos minutos de caminhada para acesso aos } \\
\text { bValores } 1 \text { a } 4 \text {, onde: } 1 \text { - discordo totalmente, } 2 \text { - } \\
\text { 'Valores de } 1 \text { a 4, onde: } 1 \text { - muito insatisfeito, } 2 \text { - } \\
\text { dVariáveis utilizadas para formar a variável Satisfa } \\
\text { eValores de } 1 \text { a } 5 \text {, onde: } 1 \text { - muito insatisfeito, } 2 \text { - i } \\
\text { *A variável Satisfação bairro (índice) foi utilizada }\end{array}$ & $\begin{array}{l}\text { s de desloc } \\
\text { arte, } 3 \text { - con } \\
\text { - satisfeito } € \\
\text { dice). } \\
\text { - nem insati }\end{array}$ & $\begin{array}{l}\text { lento em u } \\
\text { rdo em pa } \\
\text { - muito sa } \\
\text { eito nem sa }\end{array}$ & $\begin{array}{l}\text { semana. } \\
4 \text { - conc } \\
\text { ito. }\end{array}$ & almente. & \\
\hline
\end{tabular}


A sexta parte do questionário abordou a percepção dos entrevistados em relação ao bairro de residência e estava subdividida em cinco seções, (i) Acesso a serviços; (ii) Características do bairro; (iii) Segurança no trânsito; (iv) Criminalidade no bairro e (v) Satisfação com o bairro. As seções de i a iv continham respostas de concordância com as afirmações apresentadas no questionário baseadas em uma escala Likert de quatro níveis: (1) discordo totalmente, (2) discordo em parte, (3) concordo em parte ou (4) concordo totalmente. As respostas para a seção v foram fornecidas em uma escala de quatro níveis de satisfação: (1) muito insatisfeito, (2) insatisfeito, (3) satisfeito e (4) muito satisfeito. A sétima parte foi referente à satisfação dos entrevistados com a qualidade de vida, contemplando o conceito de bem-estar. Os entrevistados foram questionados sobre a satisfação geral em relação à qualidade de vida e sobre aspectos específicos como saúde, relações pessoais, etc. As respostas foram baseadas em uma escala de Likert com cinco níveis de satisfação: (1) muito insatisfeito, (2) insatisfeito, (3) nem insatisfeito nem satisfeito, (4) satisfeito e (5) muito satisfeito. 0 questionário completo pode ser consultado no trabalho realizado por Samios (2018). A Tabela 1 apresenta a estatística descritiva da amostra $(\mathrm{N}=2300)$ para as variáveis utilizadas.

\subsection{Classificação dos entrevistados por modo de deslocamento}

Para analisar possíveis diferenças entre usuários de TA, TC e TI na percepção do ambiente construído e a relação com o bem-estar, os dados referentes aos hábitos semanais de deslocamento relatados pelos entrevistados (frequência e duração das viagens semanais - Tabela 1), foram utilizados para classificá-los conforme o principal modo de transporte. 0 fluxograma apresentado na Figura 2 sintetiza os passos para a classificação dos entrevistados.

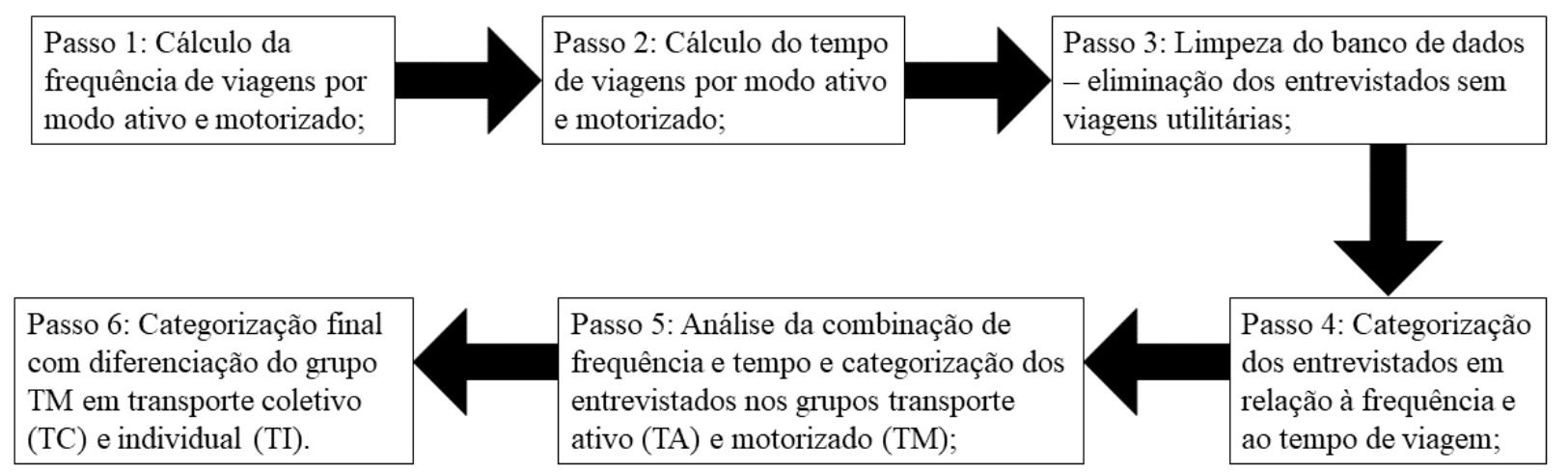

Figura 2. Fluxograma dos passos para classificação dos entrevistados

A classificação resultou em 651 usuários de TA, 633 de TC e 761 de TI; 16 pessoas classificadas como usuárias de transporte motorizado não puderam ser diferenciadas entre TC ou TI. Das 2300 entrevistas válidas, 239 foram realizadas com pessoas que não efetuaram deslocamentos e assim não foram consideradas para este estudo. A amostra final resultou em 2045 pessoas.

\section{MÉTODO}

\subsection{Modelo de equações estruturais}

O método de modelos de equações estruturais (SEM - Structured Equation Modeling) foi utilizado para analisar simultaneamente as inter-relações entre a percepção de aspectos do ambi- 
ente construído e o bem-estar para cada um dos grupos. Modelos de equações estruturais permitem analisar conceitos ou variáveis que não podem ser medidas diretamente, chamadas de construtos ou variáveis latentes. Nos modelos SEM, múltiplos indicadores ou variáveis observadas são usados para determinar as variáveis latentes, e representam uma teoria, que pode ser baseada na prática, por experiência ou observação do comportamento real. 0 papel da modelagem estrutural é confirmar tal teoria, visto que as relações são especificadas pelo pesquisador antes que o modelo possa ser estimado (Hair et al., 2009; Schumacker e Lomax, 2010).

0 modelo SEM é formado por dois componentes que representam (i) a teoria do modelo de mensuração e (ii) a teoria do modelo estrutural. 0 modelo de mensuração é estabelecido em um passo anterior ao modelo estrutural, pois nele são especificados os indicadores que possuem relações causais com a variável latente. 0 modelo de mensuração é avaliado para estabelecer a confiabilidade da formação da variável latente. 0 segundo componente especifica como as variáveis latentes em um modelo são relacionadas (Hair et al., 2009). Os mesmos índices de ajuste são utilizados para a validação dos dois modelos.

A elaboração do modelo proposto neste trabalho foi baseada em estudos prévios desenvolvidos pelos autores (Larranaga et al., 2014; Larranaga e Cybis, 2014; Lucchesi et al., 2018; Samios, 2018), assim como em técnicas multivariadas. Análises prévias foram realizadas para a construção do modelo SEM, adotando-se etapas indicadas na literatura (Hair et al., 2009). Análises de correlações foram utilizadas para explorar possíveis associações entre variáveis observadas na construção das variáveis latentes. Adotou-se a etapa de análise fatorial exploratória (AFE) para identificar o número de variáveis latentes que melhor representaria as variáveis observadas. A análise fatorial confirmatória (AFC) foi utilizada em uma etapa posterior para testar a validade da teoria de formação das variáveis latentes e compreender a avaliação do modelo de mensuração. A partir destas análises definiu-se o modelo de equações estruturais final, o qual foi estimado através do método dos mínimos quadrados generalizados, utilizando o programa computacional IBM SPSS Amos 22. Técnicas de reamostragem Bollen-Stine Bootstrap foram utilizadas para eliminar erros de não normalidade (Kim e Millsap, 2014).

\subsubsection{Modelo de mensuração}

O modelo de mensuração foi constituído por duas variáveis latentes: (i) Segurança Pública e (ii) Caminhabilidade. A primeira representa a percepção de segurança pública no bairro e é composta pelas variáveis observadas obtidas através de perguntas do questionário correspondentes a seção "Criminalidade no bairro". Estas perguntas se referem à segurança para caminhar ou andar de bicicleta de dia (Seguro_TA_dia), à noite (Seguro_TA_noite), para embarcar ou desembarcar do transporte público (Seguro_embar_desembar_TC) e para frequentar locais públicos de lazer (Seguro_parq_espaç_publicos).

A variável latente Caminhabilidade agrupa as demais características do ambiente construído que se referem à conveniência e atratividade do ambiente construído para a realização de viagens a pé. Estas variáveis representam os seguintes componentes: (i) desenho urbano, (ii) qualidade do ambiente e (iii) infraestrutura para pedestres. As etapas adotadas para a definição do modelo de mensuração são descritas com maiores detalhes em estudo anterior (Samios, 2018). Os parâmetros estimados para as variáveis observadas utilizadas no modelo de mensuração tiveram a sua significância estatística atestada para os três grupos através de valores de CR e valor-p. As variáveis Coisas_interessantes_ver e Seguro_parq_espaços_públicos tiveram suas 
escalas fixadas para possibilitar a identificação do modelo, essas variáveis foram escolhidas pela premissa de possuírem relação positiva com as respectivas variáveis latentes.

As duas variáveis latentes possuem características distintas. Segurança Pública foi modelada de forma reflexiva, indicando que esta variável se manifesta ou reflete nas variáveis observadas, as quais apresentam correlações médias e altas (coeficiente de correlação de Pearson entre 0.3 e 0.7) (Hair et al., 2009). Caminhabilidade foi modelada de maneira formativa, pois ela é formada pelas variáveis observadas. Neste caso, não se espera correlação entre as variáveis observadas (Diamantopoulos et al., 2008).

\subsubsection{0 modelo estrutural}

A teoria estrutural do modelo foi representada pela relação biunívoca entre Caminhabilidade e Segurança Pública. Esta relação considera que a caminhabilidade do bairro pode afetar a percepção de segurança pública, pois ao incentivar mais viagens a pé proporciona vivacidade e aprazibilidade às ruas do bairro, assim como a segurança pública pode afetar a caminhabilidade, visto que a sensação de insegurança pode inibir os deslocamentos a pé prejudicando a caminhabilidade do ambiente urbano (Jacobs, 2011; Lucchesi et al., 2018). 0 modelo de equações estruturais proposto adicionou a satisfação com o bairro (Satisfação_bairro) e o bem-estar (Bem-estar) como variáveis observadas endógenas (Hair et al., 2009).

Satisfação_bairro foi incluída como uma variável mediadora entre o ambiente construído e o bem-estar. A relação entre ambiente construído e satisfação com o bairro já foi relatada por estudos anteriores (Lee et al., 2017; Kim et al., 2014; Grasser et al., 2016). Satisfação_bairro foi criada como um indicador da satisfação geral com o bairro para cada entrevistado, e considera a média das respostas relativas às oito perguntas de satisfação com diversas características do bairro, incluídas na sexta seção do questionário e apresentadas na Tabela 1.0 procedimento relativamente comum de médias aritméticas foi utilizado como indicador da satisfação, mesmo sabendo-se que idealmente deveriam ser usadas frequências para variáveis categóricas.

Através da variável mediadora foi proposta a relação entre ambiente construído e bem-estar. O modelo SEM proposto visou analisar a percepção de características do ambiente urbano que influenciam a caminhabilidade e sua relação com o bem-estar, medido como a satisfação com a qualidade de vida. A Figura 3 apresenta o diagrama de caminhos do modelo.

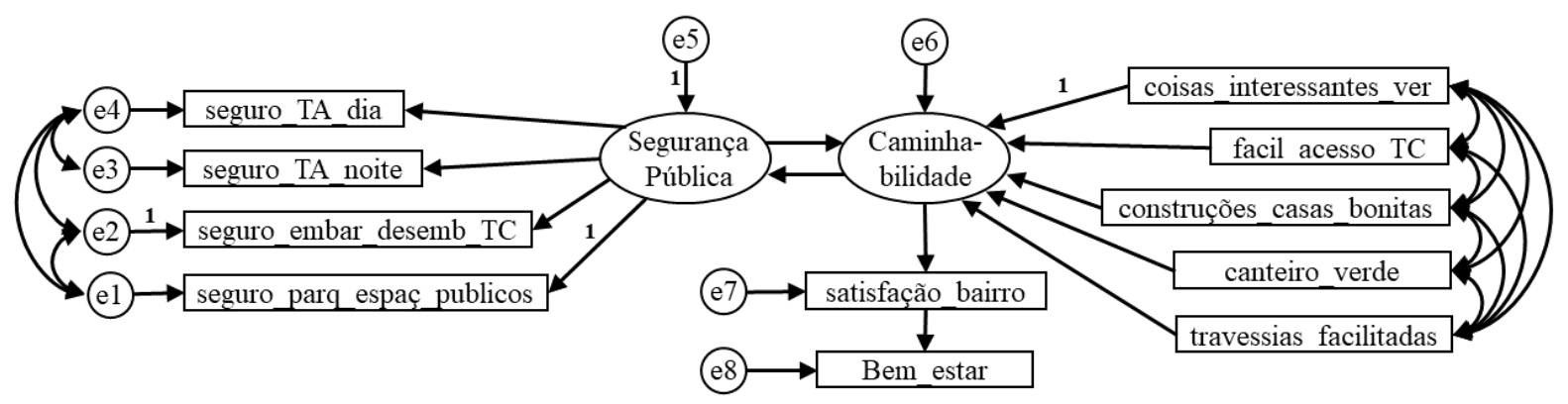

Figura 3. Diagrama de caminhos do modelo SEM proposto

\subsection{Análise multigrupo}

O modelo SEM proposto foi estimado simultaneamente para os três grupos de usuários (TA, TC e TI). Para permitir a comparação entre os resultados obtidos para os três grupos e identificar 
se existiam diferenças significativas, a técnica de análise fatorial confirmatória multigrupo (AFCMG) foi utilizada. Esta é uma técnica da modelagem de equações estruturais que avalia em que medida a configuração e os parâmetros de determinado modelo são invariantes (equivalentes) para diferentes grupos (Brown et al., 2015; Limbers et al., 2009; Her et al., 2018; Hair et al., 2009).

Através da comparação de um modelo restrito e outro irrestrito, a equivalência ou invariância entre os grupos foi testada e determinada examinando-se as alterações na qualidade de ajuste dos modelos. Realizou-se inicialmente a estimação de um modelo irrestrito, onde os parâmetros correspondentes entre os grupos foram livremente calculados, e posteriormente de um modelo restrito, ao qual foi imposta a restrição de igualdade para os parâmetros correspondentes entre os grupos. A avaliação da invariância entre os modelos estimados, com e sem restrições, foi realizada utilizando o teste de diferença do qui-quadrado $\left(\Delta \chi^{2}\right)$, o mais utilizado na literatura para AFCMG (Cheung e Rensvold, 2002; Hair et al., 2009). A obtenção de uma diferença estatisticamente significativa $\left(\Delta \chi^{2}<0,05\right.$, para $95 \%$ de confiança $)$ indica que o pressuposto de invariância não é aceito, possibilitando a comparação dos resultados obtidos para os grupos analisados (Cheung e Rensvold, 2002).

\section{RESULTADOS E ANÁLISE}

A Tabela 2 apresenta os resultados e os parâmetros estimados para os três grupos de usuários para o modelo sem restrições. São apresentados os valores dos pesos padronizados (P.E) dos parâmetros, assim como o valor-p que atesta a significância estatística dos parâmetros calculados para as variáveis presentes no modelo (Hair et al., 2009). 0 modelo apresentou índices de ajuste satisfatórios, indicando boa adequação aos dados. GFI igual a 0.95 (superior a 0.9) e RMSEA igual a 0.51 (menor que 0.60 ), os valores de referência são os recomendados na literatura (Hair et al., 2009). 0 teste de $\Delta \chi^{2}$ foi significativo para $95 \%$ de confiança, rejeitando a hipótese de invariância entre os grupos analisados, sendo possível afirmar que existe diferença entre os usuários de TA, TC e TI.

Tabela 2 - Resultados da estimação do modelo multigrupo SEM - modelo sem restrições

\begin{tabular}{|c|c|c|c|c|c|c|c|c|}
\hline \multirow{2}{*}{\multicolumn{3}{|c|}{ Relações Causais }} & \multicolumn{2}{|c|}{ Grupo TA } & \multicolumn{2}{|c|}{ Grupo TI } & \multicolumn{2}{|c|}{ Grupo TC } \\
\hline & & & \multirow{2}{*}{\begin{tabular}{|c|} 
P. E. \\
0,121
\end{tabular}} & \multirow{2}{*}{$\begin{array}{c}P^{*} \\
0,003\end{array}$} & \multirow{2}{*}{\begin{tabular}{|c|} 
P. E. \\
0,171 \\
\end{tabular}} & \multirow{2}{*}{$\begin{array}{c}P^{*} \\
0,008\end{array}$} & \multirow{2}{*}{$\begin{array}{c}\text { P. E. } \\
0,134\end{array}$} & \multirow{2}{*}{$\begin{array}{c}P^{*} \\
0,012\end{array}$} \\
\hline Caminhabilidade & $<-$ & Construções_casas_bonitas & & & & & & \\
\hline Caminhabilidade & $<-$ & Fácil_acesso_TC & 0,151 & $* * *$ & 0,130 & 0,006 & 0,099 & 0,01 \\
\hline Caminhabilidade & $<-$ & Canteiro_verde & 0,207 & $* * *$ & 0,319 & 0,003 & 0,271 & 0,001 \\
\hline Caminhabilidade & $<-$ & Travessias_facilitadas & 0,237 & $* * *$ & 0,226 & 0,002 & 0,126 & 0,015 \\
\hline Caminhabilidade & $<-$ & Coisas_interessantes_ver & 0,125 & - & 0,114 & - & 0,136 & - \\
\hline Satisfação_bairro & $<-$ & Caminhabilidade & 0,775 & $* * *$ & 0,803 & $* * *$ & 0,833 & $* * *$ \\
\hline Seguro_parq_espaç_publicos & $<-$ & Segurança Pública & 0,593 & - & 0,625 & - & 0,607 & - \\
\hline Seguro_emb_desembar_TC & $<-$ & Segurança Pública & 0,575 & $* * *$ & 0,514 & $* * *$ & 0,345 & $* * *$ \\
\hline Seguro_TA_noite & $<-$ & Segurança Pública & 0,619 & $* * *$ & 0,646 & $* * *$ & 0,59 & $* * *$ \\
\hline Seguro_TA_dia & $<-$ & Segurança Pública & 0,462 & $* * *$ & 0,247 & $* * *$ & 0,253 & $* * *$ \\
\hline Bem_estar & $<-$ & Satisfação_bairro & 0,337 & $* * *$ & 0,267 & $* * *$ & 0,304 & $* * *$ \\
\hline Segurança Pública & $<-$ & Caminhabilidade & 0,678 & $* * *$ & 0,453 & $* * *$ & 0,506 & $* * *$ \\
\hline Caminhabilidade & $<-$ & Segurança Pública & 0,573 & 0,002 & 0,544 & 0,002 & 0,638 & 0,004 \\
\hline
\end{tabular}


Tabela 2 - Resultados da estimação do modelo multigrupo SEM - modelo sem restrições (continuação)

\begin{tabular}{c|c|c|c}
\hline Relações Causais & Grupo TA & Grupo TI & Grupo TC \\
\cline { 2 - 4 } & P. E. P* P. E. P* & P. E. \\
\hline Número de parâmetros estimados** & P* \\
Graus de liberdade (df)** & \multicolumn{3}{|c}{108} \\
GFI* & \multicolumn{2}{|c}{90} \\
RMSEA** & \multicolumn{2}{|c}{0,95} \\
\hline
\end{tabular}

*Valor-p (bicaudal) máximo igual a 0.05 para significância estatística.

**Valores para todo o modelo que inclui os três grupos.

***Valores inferiores a 0,001.

- Variáveis com escala fixada previamente no modelo para referência, valor-p não estabelecido.

\subsection{Discussão}

Os resultados do modelo proposto confirmaram a teoria estabelecida para as relações entre variáveis latentes e observadas para todos os grupos de usuários. Os resultados permitem afirmar que a satisfação com o bairro possui um efeito direto na satisfação com o bem-estar e corroboram estudos realizados anteriormente (Sirgy e Cornwell, 2002; Dong e Qin, 2017). A análise das estimativas padronizadas para os três grupos permite observar que, para os usuários de TA, o impacto da satisfação com o bairro no bem-estar é levemente superior, mas para os três grupos um aumento na satisfação com o bairro representa um incremento significativo na satisfação pessoal com a qualidade de vida, ou seja, no bem-estar. Através das relações estabelecidas no modelo, a percepção das características da estrutura urbana, representadas por Caminhabilidade, apresenta um efeito positivo indireto no bem-estar, intermediado pela satisfação com o bairro.

Para os três grupos foi verificada a relação entre estrutura urbana (Caminhabilidade) e a satisfação com o bairro e as características percebidas do ambiente construído estão positivamente relacionadas com a satisfação com o bairro. Fatores como a atratividade do entorno, presença de vegetação, conectividade viária, infraestrutura qualificada para pedestres e segurança pública aumentam a satisfação com o bairro, bem como em estudos encontrados na literatura (Grasser et al., 2016; Leslie e Cerin, 2008; Mccrea et al.,2005; Van Dyck et al., 2011).

A comparação entre as variáveis que influenciam Caminhabilidade mostra que a Segurança Pública é a variável mais influente nos três grupos. 0 resultado enfatiza a preocupação com a segurança no entorno, principalmente em um país que apresenta altas taxas de violência e criminalidade e replica estudos anteriores (Larranaga et al., 2009; Lucchesi, 2016). A análise dos outros indicadores que influenciam na caminhabilidade aponta diferenças entre os grupos de usuários de transporte. Para o grupo usuário de TA, a oportunidade de travessias (Travessias_facilitadas) é o segundo elemento mais importante, no entanto para os outros dois grupos - TC e $\mathrm{TI}$, a variável que reflete a presença de vegetação em canteiros que separam a calçada das vias de tráfego (Canteiros_verdes) é o segundo elemento mais impactante.

Os usuários do TA apresentam maior exposição ao risco de atropelamentos, sendo usuários mais vulneráveis. A conveniência de travessia, seja por faixas de pedestres, sinalização semafórica específica ou por passarelas, aparenta ser um fator especialmente importante para estes usuários trafegarem com segurança. É possível que usuários de modos motorizados não tenham a mesma percepção desse risco, pois não realizam viagens a pé com a mesma frequência. A região de estudo possui uma importante barreira física que dificulta a conexão a pé entre a porção 
norte e sul da área. A Avenida Radial Leste, assim como a linha de metrô em superfície, dificilmente podem ser transpostas por pedestres sem a existência de infraestrutura adequada para travessia. A presença de vegetação nas calçadas pode estar atrelada à atratividade da área, tornando-a mais convidativa e confortável, ou também como um elemento de separação que confere maior comodidade ao pedestre. Os grupos apresentam ordens diferentes quantos aos demais indicadores que influenciam na caminhabilidade, sendo interessante analisar quais indicadores são mais importantes para cada grupo no momento de traçar estratégias para a melhoria da percepção da caminhabilidade na região.

Para os três grupos, as variáveis referentes à segurança para caminhar à noite (Seguro_TA_noite) e para frequentar espaços públicos (Seguro_espaços_públicos) são as mais impactadas por um aumento na percepção de segurança pública em geral. Esses resultados são condizentes com teorias de espaço urbano que explicam a importância da vitalidade urbana para uma maior percepção de segurança (Gehl, 2013; Jacobs, 2011).

A comparação da relação biunívoca entre as variáveis latentes, Segurança Pública e Caminhabilidade, mostram resultados distintos para os grupos ativos e motorizados. Para os usuários de TA, o maior impacto nesta relação corresponde ao impacto da Caminhabilidade na Segurança Pública. Enquanto que, para os dois outros grupos - TC e TI, a relação é inversa. Tal resultado indica que os indivíduos que não caminham regularmente para realizar atividades diárias, parecem superestimar a preocupação com a segurança pública. 0 motivo pode ser atribuído a um fenômeno crônico no Brasil, relacionado com a generalização da sensação de insegurança nas ruas, possivelmente maior do que a insegurança real. Caminhantes regulares atribuíram maior importância para elementos concretos da sua experiência real de caminhar, percebendo com mais clareza detalhes da estrutura urbana e da morfologia arquitetônica. Os indivíduos adeptos aos modos de transportes motorizados tendem a ser mais abstratos, recorrendo a critérios mais genéricos e informações indiretas a respeito da qualidade ambiental para realizar deslocamentos a pé (Vargas et al., 2016).

Os resultados do modelo mostram que o ambiente construído pode influenciar o bem-estar dos indivíduos. A percepção de segurança pública é a principal característica do ambiente urbano com potencial de impactar o bem-estar. Para os usuários de TA, um ambiente construído que facilite e dê segurança aos deslocamentos (Travessias_facilitadas) é a segunda característica com maior impacto no bem-estar, enfatizando que medidas de desenho viário que priorizem os deslocamentos ativos podem, além de incentivar a caminhabilidade e melhorar a segurança viária, melhorar a avaliação pessoal de qualidade de vida. A presença de vegetação nas calçadas mostrou-se o segundo elemento a impactar o bem-estar para os usuários de transportes motorizados, e o terceiro para usuários de TA. As demais características do ambiente construído consideradas nesse estudo impactam de forma similar os três grupos, apresentando pesos semelhantes, porém se confirmam como características do ambiente construído com potencial de influenciar positivamente o bem-estar da população.

Os resultados enfatizam que as formas urbanas caracterizadas por uso do solo heterogêneo, que possibilitam caminhos menos monótonos e com maior diversidade de destinos e atividades, com condições estéticas agradáveis, arborizadas, que facilitem o acesso ao transporte coletivo e são orientadas ao deslocamento ativo podem impactar positivamente o bem-estar das pessoas. Estes resultados corroboram estudos anteriores como os realizados por Mouratidis, (2018), Leyden, (2003) e Wood et al., (2010). Os resultados auxiliam na discussão e elaboração 
de estratégias de planejamento urbano que busquem, além da qualificação da mobilidade, a melhoria da qualidade de vida e bem-estar da população.

\section{CONSIDERAÇÕES FINAIS}

Este trabalho explorou a relação entre estrutura urbana e bem-estar, analisando a existência de diferenças entre usuários de transporte ativo, transporte coletivo e transporte individual com residentes de bairros da zona leste de São Paulo. 0 estudo desenvolvido mostrou que o ambiente construído influencia o bem-estar dos indivíduos para os três grupos de usuários de transporte. Características como a segurança pública, medidas que facilitam e melhoram a segurança viária dos pedestres, presença de vegetação em canteiros e calçadas, uso misto do solo e atratividade impactaram positivamente o bem-estar pessoal para os três grupos analisados.

Os resultados mostraram a existência de diferenças na percepção do ambiente construído entre usuários de transporte ativo, coletivo e individual. As diferenças principais foram encontradas entre os usuários de transporte ativo e os usuários de transporte motorizado (coletivo e individual), tanto em relação à percepção do ambiente, quanto à sua influência no bem-estar. A semelhança entre usuários de transporte motorizado indica que o modo habitual de deslocamento pode ser um fator importante para explicar o bem-estar.

Em relação ao impacto da percepção de segurança pública na caminhabilidade, maior importância aos aspectos concretos da experiência de caminhar foi atribuída pelas pessoas que realizam caminhadas utilitárias regularmente. A segurança pública parece ser superestimada por aqueles que não realizam deslocamentos ativos frequentes e optam por modos motorizados para deslocamentos diários. Usuários ativos também atribuíram maior importância a elementos que podem reduzir a exposição ao risco de acidentes de tráfego, enquanto características estéticas do entorno, como a presença de vegetação, impactaram mais a caminhabilidade para os dois grupos de usuários de transporte motorizado. Os resultados demonstram que as medidas que visem incrementar a segurança pública têm um importante papel no estímulo à caminhada para os três grupos, assim como reforçam a oportunidade de estímulo aos modos ativos através de mudanças na forma urbana.

Em relação à variável mediadora, referente à satisfação com o bairro, os resultados mostraram uma relação positiva para os três grupos de usuários. A satisfação com o bairro impacta diretamente o bem-estar, verificando estudos realizados anteriormente (Sirgy e Cornwell, 2002; Dong e Qin, 2017). A relação direta e positiva entre caminhabilidade e satisfação com o bairro para os três grupos possibilita afirmar que bairros caminháveis são preferíveis por todos os usuários e além dos benefícios gerados pelo estímulo aos modos sustentáveis de transporte, têm o potencial de melhorar o bem-estar pessoal. Tal resultado enfatiza a importância de se estabelecer estratégias de planejamento urbano que priorizem cidades mais ativas e que estimulam modos de deslocamento sustentáveis para melhorar a qualidade de vida da população.

Os resultados obtidos entre ambiente construído e bem-estar dos indivíduos podem contribuir para debates acadêmicos e políticos sobre bem-estar, qualidade de vida e sustentabilidade urbana considerando a falta de investigações empíricas aprofundadas sobre este tópico.

\section{AGRADECIMENTOS}

Os autores agradecem ao WRI Brasil pela disponibilização da pesquisa domiciliar utilizada e ao CNPq pelo apoio financeiro. 


\section{REFERÊNCIAS}

Basarić V.; A. Vujičić; J. M. Simić; V. Bogdanović e N. Saulić (2016) Gender and Age Differences in the Travel Behavior - A Novi Sad Case Study. Transportation Research Procedia, v. 14, p. 4324-4333. DOI:10.1016/j.trpro.2016.05.354.

Brown, G. T.; L. R. Harris; C. O'Quin e K. E. Lane (2015) Using multi-group confirmatory factor analysis to evaluate cross-cultural research: identifying and understanding non-invariance. International Journal of Research \& Method in Education. v. 40, n. 1, p. 66-90. DOI:10.1080/1743727X.2015.1070823.

Cabrera, J. F. (2013). New urbanism and selection bias in the formation of social capital. Housing Policy Debate, v. 23, n. 2, p. 376-394. DOI:10.1080/10511482.2013.766626.

Cheung, G.W. e R. B. Rensvold (2002) Evaluating goodness-of-fit indexes for testing measurement invariance. Structural equation modeling, v. 9, n. 2, p. 233-255. D0I:10.1207/S15328007SEM0902_5.

Delbosc, A. (2012) The role of well-being in transport policy. Transp. Policy, v. 23, p. 25-33. DOI:10.1016/j.tranpol.2012.06.005.

Diamantopoulos, A.; P. Riefler e K. P. Roth (2008) Advancing formative measurement models. Journal of Business Research, v. 61, n. 12, p. 1203-1218. DOI:10.1016/j.jbusres.2008.01.009.

Diener, E. (1984) Subjective Well-Being. Psychological Bulletin, v. 95, p. 542-575. DOI:10.1037/0033-2909.95.3.542.

Diener, E. (1994) Assessing subjective wellbeing: progress and opportunities. Social Indicators Research, v. 31, p. $103-157$. DOI:10.1007/BF01207052.

Dong, H. e Qin, B. (2017) Exploring the link between neighborhood environment and mental wellbeing: A case study in Beijing, China. Landscape and Urban Planning, v. 164, p. 71-80. D0I:10.1016/j.landurbplan.2017.04.005.

Elias, W.; J. Benjamin e Y. Shiftan (2015) Gender differences in activity and travel behavior in the Arab world. Transport Policy, v. 44, p. 19-27. ISSN 0967-070X. DOI:10.1016/j.tranpol.2015.07.001.

Gehl, J. (2013) Cities for people. Island press. ISBN 9781597265737

González, R. M.; E. Martínez-Budría; J. J. Díaz-Hernández e A. Esquivel (2015) Explanatory factors of distorted perceptions of travel time in tram. Transportation Research Part F: Traffic Psychology and Behaviour, v. 30, p. 107-114. ISSN 1369-8478. DOI:10.1016/j.trf.2015.02.006.

Grasser, G.; S. Titze e W. J. Stronegger (2016) Are residents of high-walkable areas satisfied with their neighbourhood? Journal of Public Health, v. 24, n. 6, p. 469-476. DOI:10.1007/s10389-016-0744-5.

Hair, J. F.; W. C. Black; B. J. Babin; R. E. Anderson e R. L. Tatham (2009) Análise Multivariada de Dados. 6 ed. Bookman. Porto Alegre. ISBN 857780402X

Heizomi, H.; H. Allahverdipour; M. A. Jafarabadi e A. Safaian (2015) Happiness and its relation to psychological well-being of adolescents. Asian Journal of Psychiatry, v. 16, p. 55-60. DOI:10.1016/j.ajp.2015.05.03.

Her, Y. W.; H. Shin e S. Pae (2018) A multigroup SEM analysis of moderating role of task uncertainty on budgetary participation-performance relationship: Evidence from Korea. Asia Pacific Management Review. DOI:10.1016/j.apmrv.2018.02.001.

Hipp, J. R. e A. J. Perrin (2009) The simultaneous effect of social distance and physical distance on the formation of neighborhood ties. City \& Community, v. 8, n. 1, p. 5-25. DOI:10.1111/j.1540-6040.2009.01267.x.

Hu, L. (2017) Changing travel behavior of Asian immigrants in the U.S. Transportation Research Part A: Policy and Practice, v. 106, p. 248-260. ISSN 0965-8564. DOI:10.1016/j.tra.2017.09.019.

Jacobs J. (2011) Morte e vida de grandes cidades. 2. ed. [s.l.] WMF Martins Fontes. ISBN 9788578274214

Joshanloo, M. (2017) Mediators of the relationship between externality of happiness and subjective well-being. Personality and Individual Differences, v. 119, p. 147-151. DOI:10.1016/j.paid.2017.07.017.

Kim, H. e R. Millsap (2014) Using the Bollen-Stine Bootstrapping Method for Evaluating Approximate Fit Indices. NIH Public Access, v. 49, n. 6, p. 161-169. DOI:10.1080/00273171.2014.947352.

Kim, S.; S. Park e J. S. Lee (2014) Meso-or micro-scale? Environmental factors influencing pedestrian satisfaction. Transportation Research Part D: Transport and Environment, v. 30, p. 10-20, 2014. DOI:10.1016/j.trd.2014.05.005.

Larranaga, A. M. e H. B. B. Cybis (2014) The relationship between built environment and walking for different trip purposes in Porto Alegre, Brazil. Int Journal of Sustainable Development and Planning, v. 9, n. 4, p. 568-580. D0I:10.2495/SDP-V9-N4568-580.

Larranaga, A. M.; J. L. D. Ribeiro e H. B. B. Cybis (2009) Fatores que afetam as decisões individuais de realizar viagens a pé: estudo qualitativo. Transportes, v. 17, n. 2. DOI:10.14295/transportes.v17i2.355.

Larranaga, A. M.; L. I. Rizzi; J. Arellana; O. Strambi e H. B. B. Cybis (2014) The Influence of built environment and travel attitudes on walking: a case study of Porto Alegre, Brazil. International Journal of Sustainable Transportation, v. 10, n. 4, p. 332-342. DOI:10.1080/15568318.2014.933986.

Lee, S. M.; T. L. Conway; L. D. Frank; B. E. Saelens; K. L. Cain e J. F. Sallis (2017) The relation of perceived and objective environment attributes to neighborhood satisfaction. Environm and Behaviour, v. 49, n. 2, p. 136-160. DOI https://doi.org/10.1016/j.tbs.2018.02.005.

Leslie, E. e E. Cerin (2008) Are perceptions of the local environment related to neighbourhood satisfaction and mental health in adults?. Preventive Medicine, v. 47, n. 3, p. 273-278. DOI:10.1016/j.ypmed.2008.01.014.

Leyden, K. M. (2003) Social capital and the built environment: the importance of walkable neighborhoods. American Journal of Public Health, v. 93, n. 9, p. 1546-1551. DOI:10.2105/AJPH.93.9.1546. 
Limbers, C. A.; D. A. Newman e J. W. Varni (2009) Factorial Invariance of Child Self-Report Across Race/Ethnicity Groups: A Multigroup Confirmatory Factor Analysis Approach Utilizing the PedsQL ${ }^{\text {TM }} 4.0$ Generic Core Scales. Annals of Epidemiology, v. 19, n. 8, p. 575-581. DOI:10.1016/j.annepidem.2009.04.004.

Lin, T.; D. Wang e X. Guan (2017) The built environment, travel attitude, and travel behavior: Residential self-selection or residential determination?. Journal of Transport Geography, v. 65, p. 111-122. ISSN 0966-6923. DOI:10.1016/j.jtrangeo.2017.10.004.

Lucchesi, S. T; A. M. Larranaga e H. B. B. Cybis (2018) Does Walkability Raise Real Estate Values in Brazil? A Hedonic Model Approach on Rio de Janeiro City. Transportation Research Board 97th Annual Meeting. 2018 Jan 7-11; Washington DC, United States.

Lucchesi, S. T. (2016) Aplicação de preços hedônicos para avaliação da influência da caminhabilidade no preço dos imóveis. Dissertação de Mestrado - PPGEP, UFRGS. Disponível em http://hdl.handle.net/10183/150501. (Acesso em 5 agosto 2019).

Mccrea, R.; R. Stimson e J. Western (2005) Testing a moderated model of satisfaction with urban living using data for Brisbane-South East Queensland, Australia. Social Indicators Research, v. 72, n. 2, p. 121-152. DOI:10.1007/s11205-004-2211$\mathrm{x}$.

Mouratidis, K. (2018) Built environment and social well-being: How does urban form affect social life and personal relationships?. Cities, v. 74, p. 7-20. DOI:10.1016/j.cities.2017.10.020.

Musa, H. D.; M. R. Yacob; A. M. Abdullah e M. Y. Ishak (2018) Enhancing subjective well-being through strategic urban planning: Development and application of community happiness index. Sustainable Cities and Society, v. 38, p. 184-194. DOI:10.1016/j.scs.2017.12.030.

Reardon, L. e Abdallah, S. (2013) Well-being and transport: taking stock and looking forward. Transport Rev, v. 33, n. 6, p. 634657. DOI:10.1080/01441647.2013.837117.

Samios, A. A. B. (2018) Impacto do ambiente construído na utilização de modos ativos: análise das percepções dos usuários de diferentes modos de transporte. Dissertação de Mestrado - PPGEP, UFRGS. Disponível em: http://hdl.handle.net/10183/179524. (Acesso em 3 setembro 2019).

Schumacker, R. E. e R. G. Lomax (2010) A beginner's guide to structural equation modeling. Psychology press.

Singleton, P. A. (2018) Walking (and cycling) to well-being: Modal and other determinants of subjective well-being during the commute. Travel Behaviour and Society (in press). DOI:10.1016/j.tbs.2018.02.005.

Sirgy, M. J. e T. Cornwell (2002). How neighborhood features affect quality of life. Social Indicators Research, v. 59, n. 1, p. 79114. DOI:10.1023/A:1016021108513.

Spinney, J. E. L.; D. M. Scott e K. B. Newbold (2009) Transport mobility benefits and quality of life: a time-use perspective of elderly Canadians. Transport Policy, v. 16, n. 1, p. 1-11. DOI:10.1016/j.tranpol.2009.01.002.

Stanley, J. e J. Stanley (2007) Public transport and social policy goals. Road and Transport Res, v. 16, p. 20-30. ISSN 1037-5783

Stanley, J. e D. Vella-Brodrick (2009) The usefulness of social exclusion to inform social policy in transport. Transport Policy, v. 16, p. 106-114. DOI:10.1016/j.tranpol.2009.02.003.

Stanley, J.; D. A. Henscher; J. Stanley; G. Currie; W.H. Greene e D. Vella-Brodrick (2011) Social exclusion and the value of mobility. Journal of Transport Economics and Policy, v. 45, p. 197-222.

Van Dyck, D.; G. Cardon; B. Deforche e I. D. Bourdeaudhuij (2011) Do adults like living in high-walkable neighborhoods? Associations of walkability parameters with neighborhood satisfaction and possible mediators. Health \& Place, v. 17, n. 4, p. 971-977. DOI:10.1016/j.healthplace.2011.04.001.

Vargas, J. C. B.; A. M. L. Uriarte e H.B.B. Cybis (2016) Explorando as viagens a pé: estrutura urbana e sensação de segurança. Anais do XXX Congresso de Pesquisa e Ensino em Transportes, ANPET.

Wang, D. e X. Cao (2017) Impacts of the built environment on activity-travel behavior: Are there differences between public and private housing residents in Hong Kong? Transportation Research Part A: Policy and Practice, v. 103, p. 25-35. DOI:10.1016/j.tra.2017.05.018.

Wood, L.; L. D. Frank e B. Giles-Corti (2010) Sense of community and its relationship with walking and neighborhood design. Social Science \& Medicine, v. 70, n.9, p. 1381-1390. DOI:10.1016/j.socscimed.2010.01.021.

Yang, S.; Y. Fan; W. Deng e L. Cheng (2017) Do built environment effects on travel behavior differ between household members? A case study of Nanjing, China. Transport Policy. ISSN 0967-070X. DOI:10.1016/j.tranpol.2017.12.006.

Ziersch, A. M.; F. E. Baum; C. MacDougall e C. Putland (2005) Neighbourhood life and social capital: The implications for health. Social Science \& Medicine, v. 60, n. 1, p. 71-86. DOI:10.1016/j.socscimed.2004.04.027. 